Supporting Information

\title{
Post Synthesis Ligand Exchange Induced Porphyrin Hybrid Crystalloid Reconstruction for Self-Enhanced Electrochemiluminescence
}

YiXuan $\mathrm{Li}^{[\mathrm{[a}]}$, Junji Li ${ }^{*[a]}$, WenRong Cai ${ }^{[\mathrm{a}]}$, WenLi Xin ${ }^{[a]}$, Robert S. Marks ${ }^{[\mathrm{b}]}$, Hai-Bo Zeng ${ }^{[\mathrm{a}]}$, Serge Cosnier ${ }^{[\mathrm{c}]}$, XueJi Zhang ${ }^{[\mathrm{a}]}$, Dan Shan*[a]

[a] YiXuan Junji Li, WenRong Cai, WenLi Xin, Hai-Bo Zeng, XueJi Zhang, Dan Shan School of Environmental and Biological Engineering

Nanjing University of Science and Technology

Nanjing 210094, China

E-mail: danshan@njust.edu.cn junjili@njust.edu.cn

[b] Robert-S. Marks

Avram and Stella Goldstein-Goren Department of Biotechnology Engineering

Ben-Gurion University of the Negev

Beer-Sheva, Israel 84105

[c] Serge Cosnier

University of Grenoble Alpes-CNRS

DCM UMR 5250

F-38000 Grenoble, France. 


\section{Table of Contents}

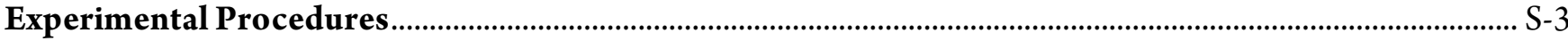

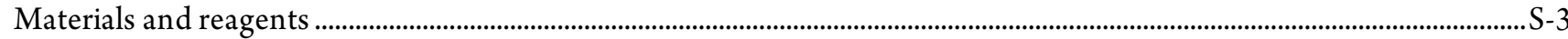

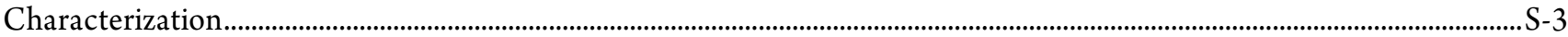

Synthesis of ZIFs-67, Co-2-MI-ZnTCPP and Co-ZnTCPP ……………………………................................................................

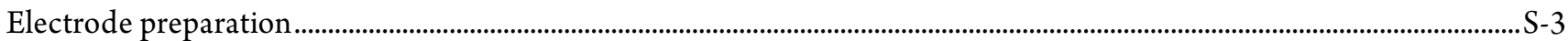

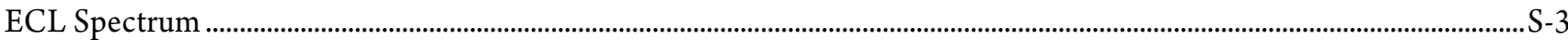

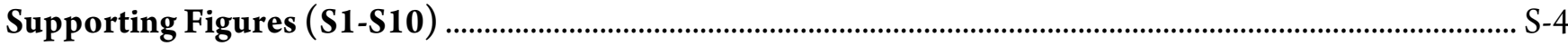




\section{Experimental Procedures}

\section{Materials and reagents}

Cobalt( II ) nitrate hexahydrate $\left(\mathrm{Co}\left(\mathrm{NO}_{3}\right)_{2} \cdot 6 \mathrm{H}_{2} \mathrm{O}, \mathrm{AR}, 99 \%\right)$, Potassium chloride (KCl), Sodium hydroxide (NaOH), 2-[4-(2-Hydroxyethyl)-1piperazinyl $]$ ethanesulfonic acid (HEPES), potassium ferricyanide $\left(\mathrm{K}_{3}\left[\mathrm{Fe}(\mathrm{CN})_{6}\right]\right)$, Potassium Ferrocyanide $\left(\mathrm{K}_{4}\left[\mathrm{Fe}(\mathrm{CN})_{6}\right]\right)$, ethanol and methanol were purchased from Aladdin (Shanghai, China). Tetraoctylammonium bromide (TOAB) were purchased from Alfa Aesar Chemicals Co. Ltd. (Shanghai, China) 2-methylimidazole (2-MI), $\left(\mathrm{C}_{4} \mathrm{H}_{5} \mathrm{~N}_{2}, \mathrm{AR}, 99 \%\right.$ ) was obtained from Maclin (Shanghai, China). Zinc(II) meso-tetra (4-carboxyphenyl) porphine (ZnTCPP) was purchased from J\&K Chemical Ltd (Shanghai, China). Ultrapure water obtained from a Millipore water purification system ( $\geq 18 \mathrm{M} \Omega$, Milli-Q Millipore) was used through the whole experiment. Electrochemiluminescence $(\mathrm{ECL})$ measurements were conducted in $10 \mathrm{mM} \mathrm{pH} 7.0 \mathrm{HEPES}$ containing $0.1 \mathrm{M} \mathrm{KCl}$ as the electrolyte.

\section{Characterization}

The graphic morphologies of the synthesized hybrid materials were obtained by using FEI Quanta250 Field Emission Scanning Electron Microscope (FESEM). Fourier-transformation infrared (FT-IR) spectra were obtained with an IR-Prestige-21 FT-IR spectrometer (Shimadzu Co., Japan). Powder X-ray diffraction patterns (PXRD) were recorded on a Bruker D8-Focus Bragg-Brentano X-ray Powder diffractometer equipped with a Cu sealed tube $(\lambda=1.54178$ $\AA ̊$ ) at room temperature. X-ray photoelectron spectroscopy (XPS) was performed by K-Alpha X-ray photoelectron spectroscopy (PHI Quantera II ESCA System). The fluorescence spectra were recorded through a fluorescence spectrophotometer (Edinburgh Analytical Instruments, FLS920) and the fluorescence spectrometer as well as a $\mathrm{CHI} 832 \mathrm{C}$ electrochemical workstation was applied to gather ECL spectra. Cyclic voltammetries (CV) were measured using a CHI 660D electrochemical workstation (CHI Co., USA). ECL measurements were performed on a MPI-E multifunctional electrochemical and chemiluminescent analytical system (Xi'an Remex Analytical Instrument Co., Ltd,China). Electrochemical impedance spectroscopy (EIS) measurements were carried out using an Autolab PGSTAT30 (Eco Chemie) controlled by NOVA 1.10 software. The electron spin resonance (ESR) were performed on a Bruker spectrometer (E500, Bruker Instrument, Germany). All electrochemical studies were carried out with a conventional three electrode system. A Pt wire electrode and a $\mathrm{Ag} / \mathrm{AgCl}$ electrode were used as counter and reference electrodes, respectively. Modified glassy carbon electrodes (GCE, $5 \mathrm{~mm}$ in diameter) were used as working electrodes.

\section{Synthesis of ZIFs-67, Co-2-MI-ZnTCPP and Co-ZnTCPP}

The proposed MOFs (Co-2-MI-ZnTCPP) were synthesized via PSE according to the protocol described as follows: $40 \mathrm{mmol} 2-\mathrm{MI}$ and $10 \mathrm{mmol}$ $\mathrm{Co}\left(\mathrm{NO}_{3}\right)_{2} \cdot 6 \mathrm{H}_{2} \mathrm{O}$ were initially dissolved in $2 \mathrm{~mL}$ and $1 \mathrm{~mL}$ methanol under sonication treatment at room temperature, respectively. The above two solutions were mixed rapidly and stirred vigorously at room temperature. $2 \mathrm{~mL}$ methanol containing $10 \mathrm{mmol}$ well-dispersed ZnTCPP was introduced $12 \mathrm{~h}$ later. The mixture was stirred vigorously for another $12 \mathrm{~h}$. The superfine green-purple powder denoted as Co-2-MI-ZnTCPP was then collected by centrifugation (4000 $\mathrm{rpm}, 10 \mathrm{~min}$ ), washed with ethanol three times, and dried in vacuum at $60^{\circ} \mathrm{C}$ for $24 \mathrm{~h}$. For the control experiment, ZIFs- 67 was prepared following a similar protocol while in the absence of ZnTCPP.

In addition, Co-ZnTCPP was also synthesized based on the previous report. Typically, $5 \mathrm{mmol} \mathrm{ZnTCPP}$ and $10 \mathrm{mmol} \mathrm{Co}\left(\mathrm{NO}_{3}\right)_{2} \cdot 6 \mathrm{H}_{2} \mathrm{O}$ were ultrasonically dissolved in $2 \mathrm{~mL}$ and $3 \mathrm{~mL}$ methanol at room temperature, respectively. The aforementioned two solutions were mixed rapidly and stirred vigorously at $80{ }^{\circ} \mathrm{C}$ for $24 \mathrm{~h}$. The purple powder of Co-ZnTCPP was harvested by centrifugation ( $4000 \mathrm{rpm}, 10 \mathrm{~min}$ ), washed with ethanol three times, and dried in vacuum at $60^{\circ} \mathrm{C}$ for $24 \mathrm{~h}$.

\section{Electrode preparation}

Initially, $1 \mathrm{mg}$ nanostructures (e.g., ZIF-67, Co-ZnTCPP and Co-2-MI-ZnTCPP) were dispersed in $100 \mu \mathrm{L}$ ethanol. Then, $10 \mu \mathrm{L}$ of the mixed solution was spread on the surface of a glassy carbon electrode (GCE). In order to enhance the stability of the electrode, after drying at ambient temperature, $10 \mu \mathrm{L}$ of a TOAB solution which was prepared by dispersing $10 \mathrm{mmol}$ of TOAB in $1 \mathrm{~mL}$ of ethanol under ultrasonication was spread on the surface of GCE.

\section{ECL Spectrum}

After $20 \mu \mathrm{L}$ of $10 \mathrm{mg} \mathrm{mL}^{-1} \mathrm{Co}-2-\mathrm{MI}-\mathrm{ZnTCPP}$ was coated at ITO electrode, the modified electrode was immersed in $10 \mathrm{mM} \mathrm{pH} 7.0$ HEPES containing $0.1 \mathrm{M} \mathrm{KCl}$ and $0.1 \mathrm{M} \mathrm{H}_{2} \mathrm{O}_{2}$ by applying a cyclic potential from $-1.7 \mathrm{~V}$ to $0 \mathrm{~V}$. 
(A)

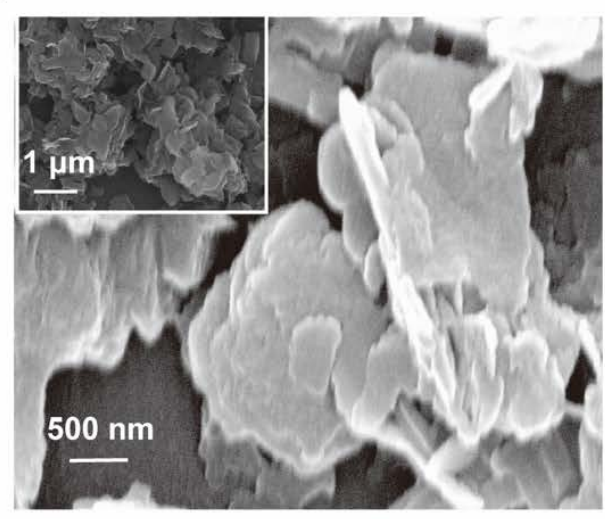

(C)

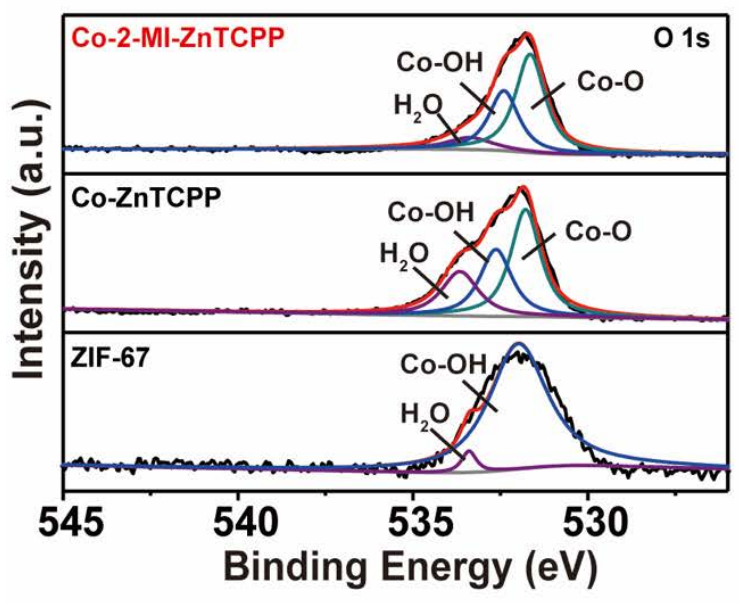

(B)

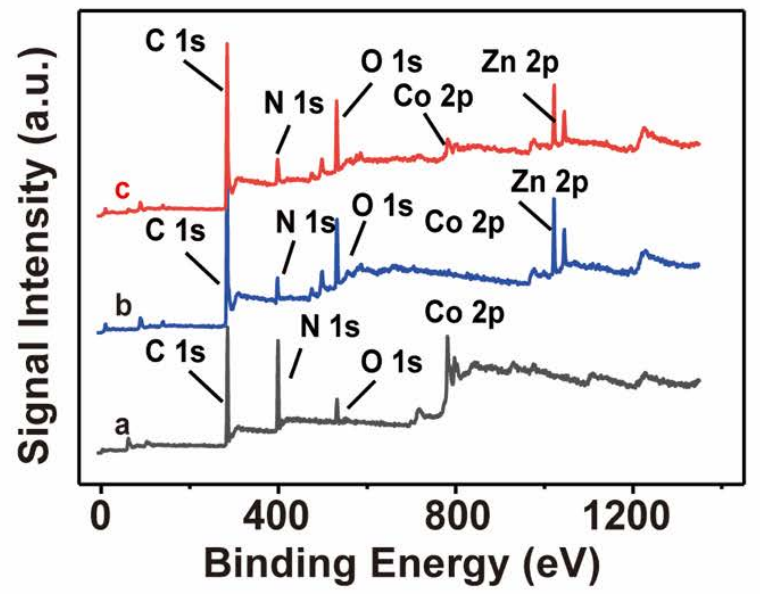

(D)

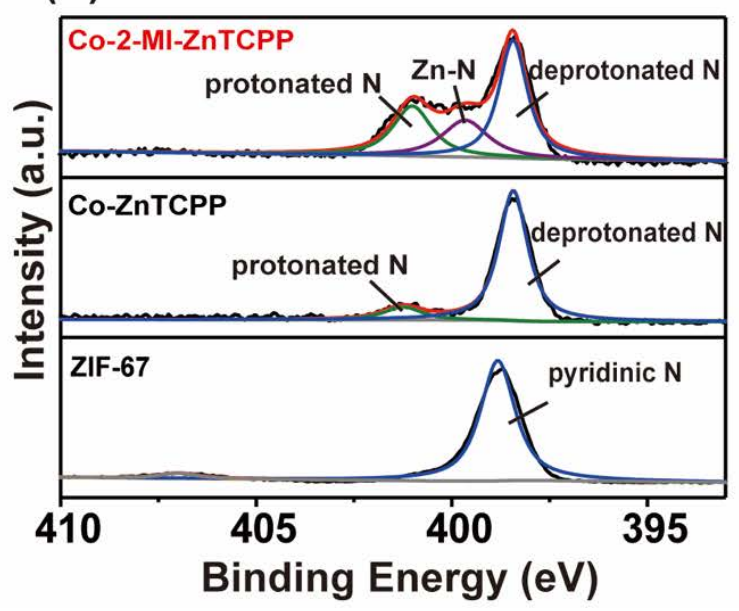

Figure S1. (A) SEM images of Co-ZnTCPP. XPS survey scan (B) and high-resolution XPS response of (C) O 1s and (D) N 1s of ZIFs-67 (a) Co-ZnTCPP (b) and Co2-MI-ZnTCPP (c).

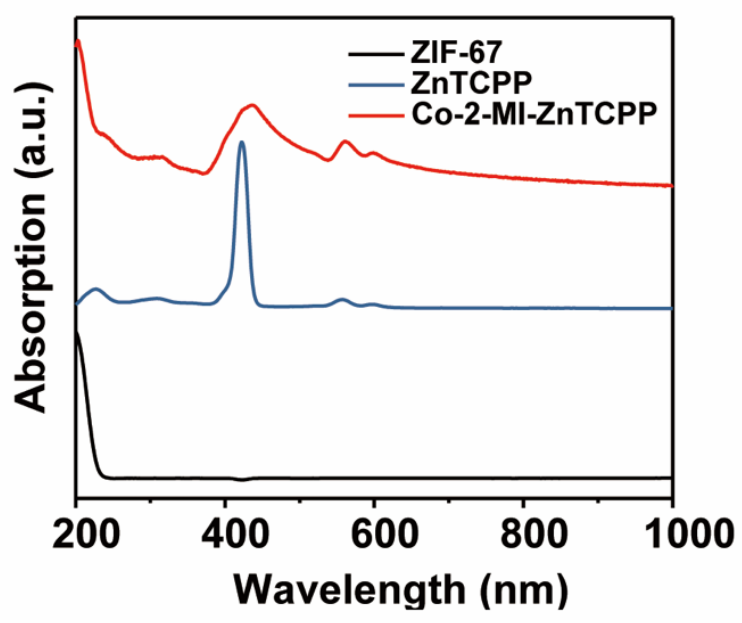

Figure S2 UV-vis absorption spectra of ZIF-67, ZnTCPP and Co-2-MI-ZnTCPP. 

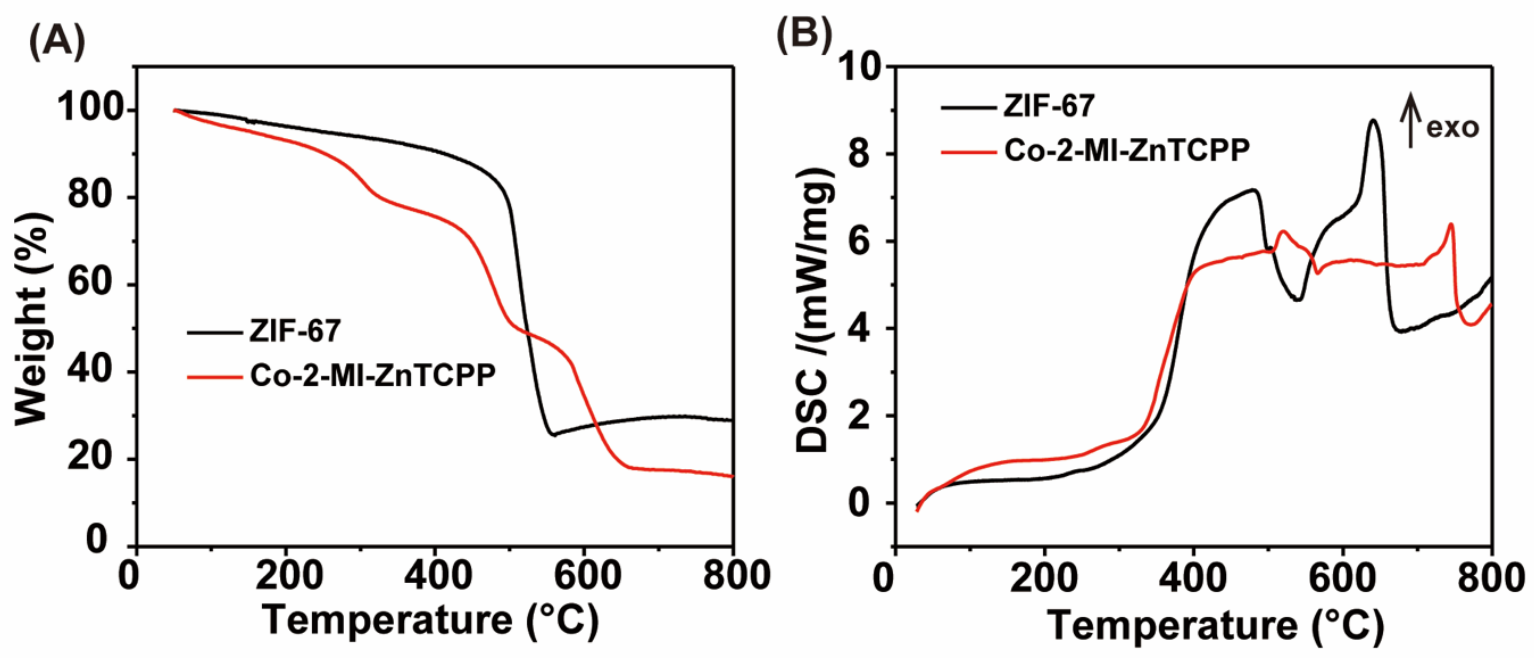

Figure S3 (A) Thermogravimetric analysis (TGA) and (B) Differential Scanning Calorimeter (DSC) in N2 flow of the ZIF-67 and Co-2-MI-ZnTCPP.

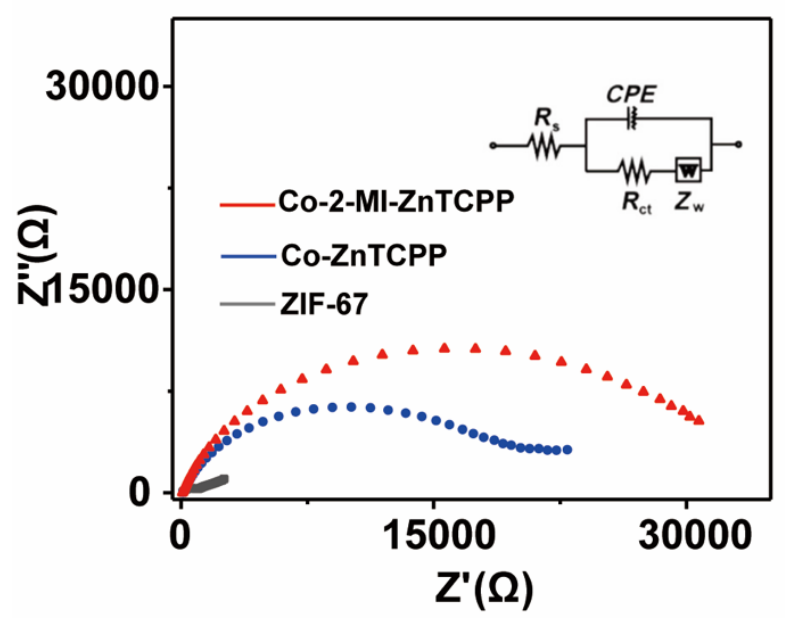

Figure S4 EIS for ZIFs-67 (a), Co-ZnTCPP (b) and Co-2-MI-ZnTCPP (c) in $0.1 \mathrm{M} \mathrm{KCl} \mathrm{solution} \mathrm{containing} 5 \mathrm{mM} \mathrm{[Fe(CN)6}]^{4-/ 3-}$.

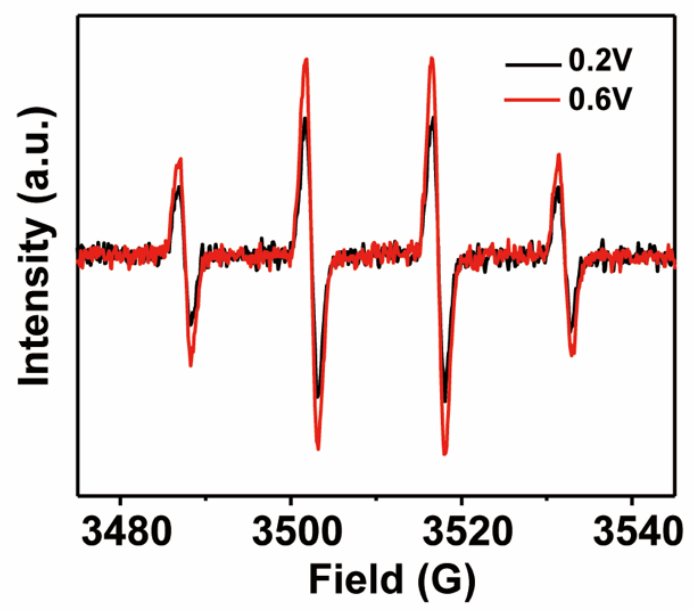

Figure S5 ESR spectrum of Co-2-MI-ZnTCPP at voltages of $-0.2 \mathrm{~V}$ and $-0.6 \mathrm{~V}$. 


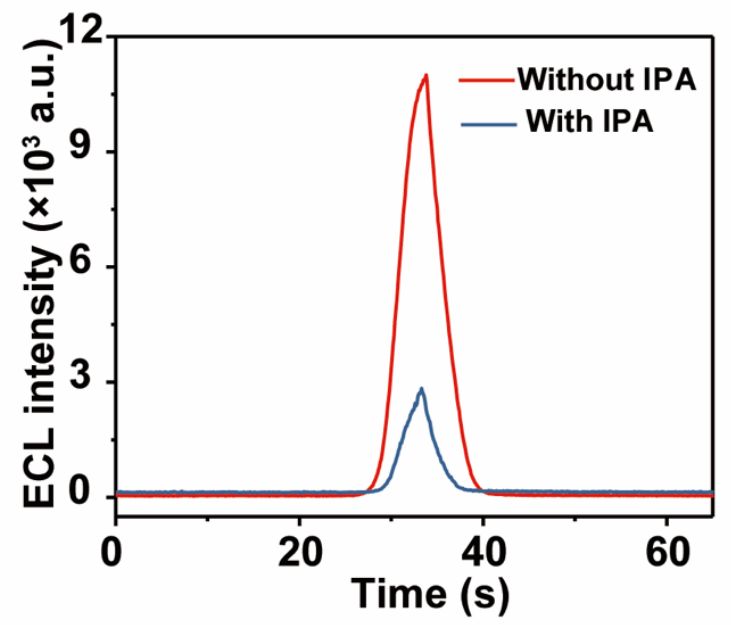

Figure S6 ECL intensity variation with addition isopropanol (IPA) as radical scavengers.

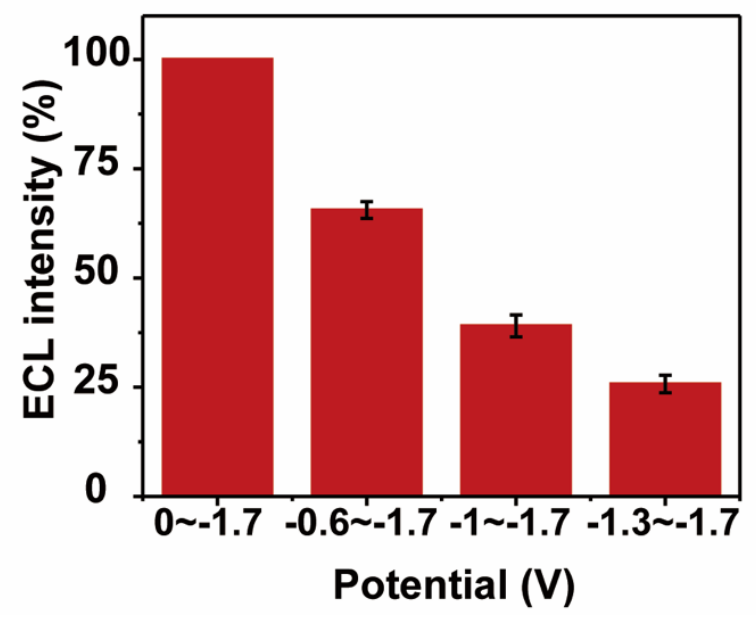

Figure S7 ECL responses of Co-2-MI-ZnTCPP in aqueous solution during a continuous potential scan between different oxidation potentials under constant sweep speed.

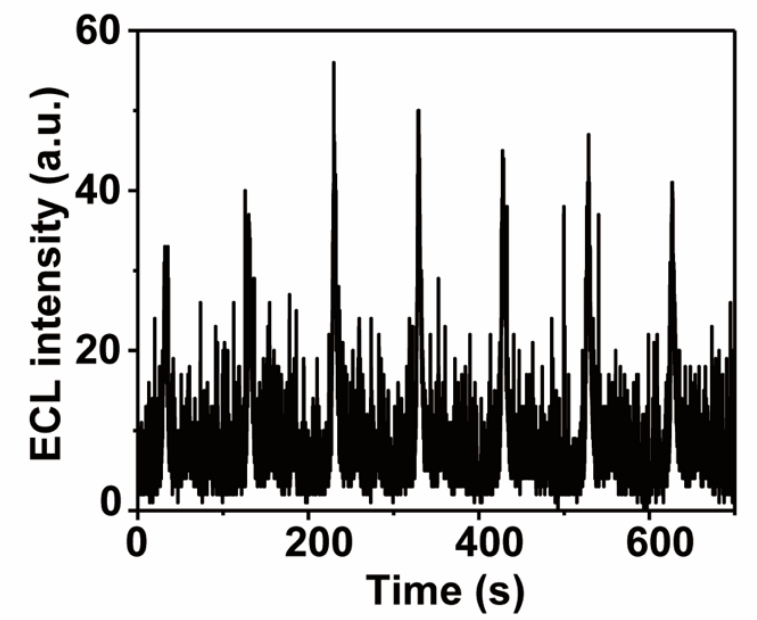

Figure S8 ECL responses of Co-2-MI-ZnTCPP in the absence of $\mathrm{H}_{2} \mathrm{O}_{2}$. 


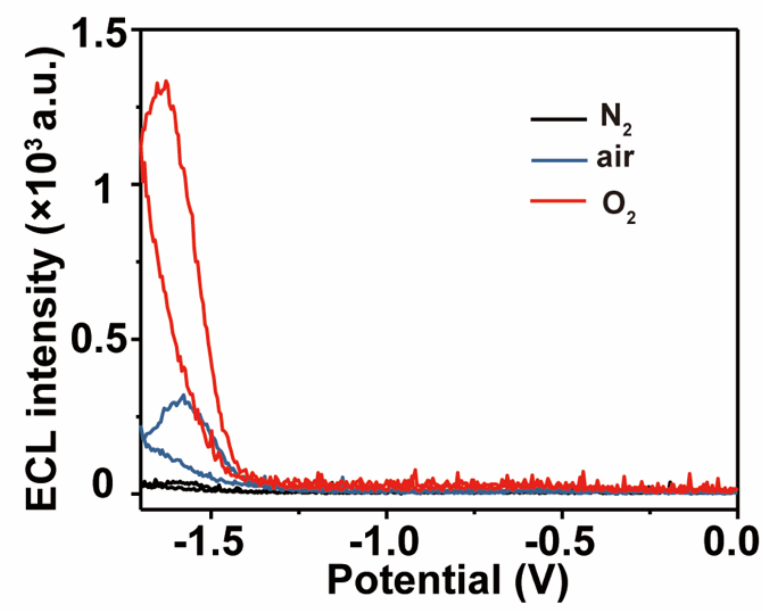

Figure S9 ECL-potential curves of Co-2-MI-ZnTCPP in aqueous solution in $\mathrm{N}_{2}$-saturated, air-saturated and $\mathrm{O}_{2}$-saturated.

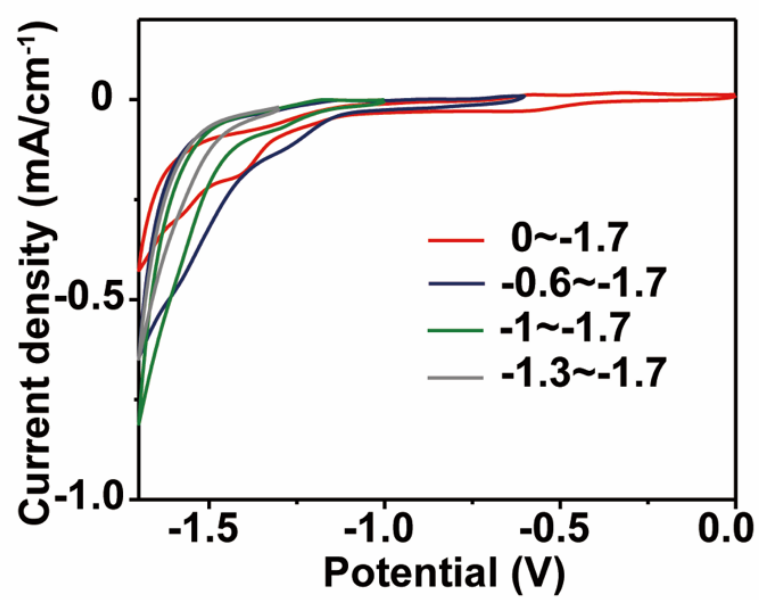

Figure S10 Cyclic voltammograms of Co-2-MI-ZnTCPP in different scanning range in the absence of $\mathrm{H}_{2} \mathrm{O}_{2}$. 